\title{
Der Hausarzt wird zum Manager des Vitamin D
}

Der Vitamin D-Spiegel hat seinen Normalwert zwischen 30 und $100 \mathrm{nmol} / \mathrm{L}$. Viele Österreicher, speziell die Älteren erreichen diese Werte nicht, bei ihnen besteht ein kleinerer oder auch ein riesengroßer Vitamin D-Mangel. Es gibt sogar Patienten mit Werten unter $10 \mathrm{nmol} / \mathrm{L}$, viele haben einen Spiegel um die 20 $\mathrm{nmol} / \mathrm{L}$, es gibt aber auch Menschen mit erfreulichen $35 \mathrm{nmol} / \mathrm{L}$. Der Blutbefund ist deshalb für Colecalciferol so wichtig wie die Kontrolle des Cholesterins. Auch die Substitution mit Vitamin D muss vom Hausarzt evaluiert werden. Ein Spiegel von $50 \mathrm{nmol} / \mathrm{L}$ scheint das individuelle Optimum zu bilden.

Wie wichtig ist das Thema Vitamin D in der Geriatrie?

Rosenberger-Spitzy: Der Vitamin D-Mangel nimmt mit dem Alter deutlich zu. In den Jahren vor dem Ableben haben fast alle Patienten starke Mangelerscheinungen. Wir wissen, dass Vitamin D die Knochen und die Muskeln schützt; es treten auch weniger Schmerzzustände auf. Es gibt einen Zusammenhang zwischen dem Vitamin D-Spiegel und der "Frailty" in den späten Lebensjahren. Frailty nennen wir die Gebrechlichkeit der Alten, die die Lebensqualität stark beeinträchtigt.

Natürlich genügt es nicht, Vitamin D zu substituieren. Wichtig ist auch das Spazierengehen draußen in der Natur und moderater Sport. Krafttraining bis ins hohe Alter ist heute ein Muss für die Prävention genauso wie tägliche Gymnastik. Die Sendereihe „Fit mit Philipp“ zählt zu den großartigsten Erfin- dungen des ORF. Täglich bewegen bis zu 250.000 Mitturner eine große Zahl ihrer 650 verschiedenen Muskeln - die Medien unterstützen damit die moderne Medizin. Ich bin selbst in meinem Wohnzimmer jeden Tag dabei!

Korreliert Frailty mit der Sturzgefahr? Ja, ganz klar. Das ist genau dieser fatale Karriereweg der älteren Menschen. Der Patient wird frail, damit stürzt er und der Sturz führt zu

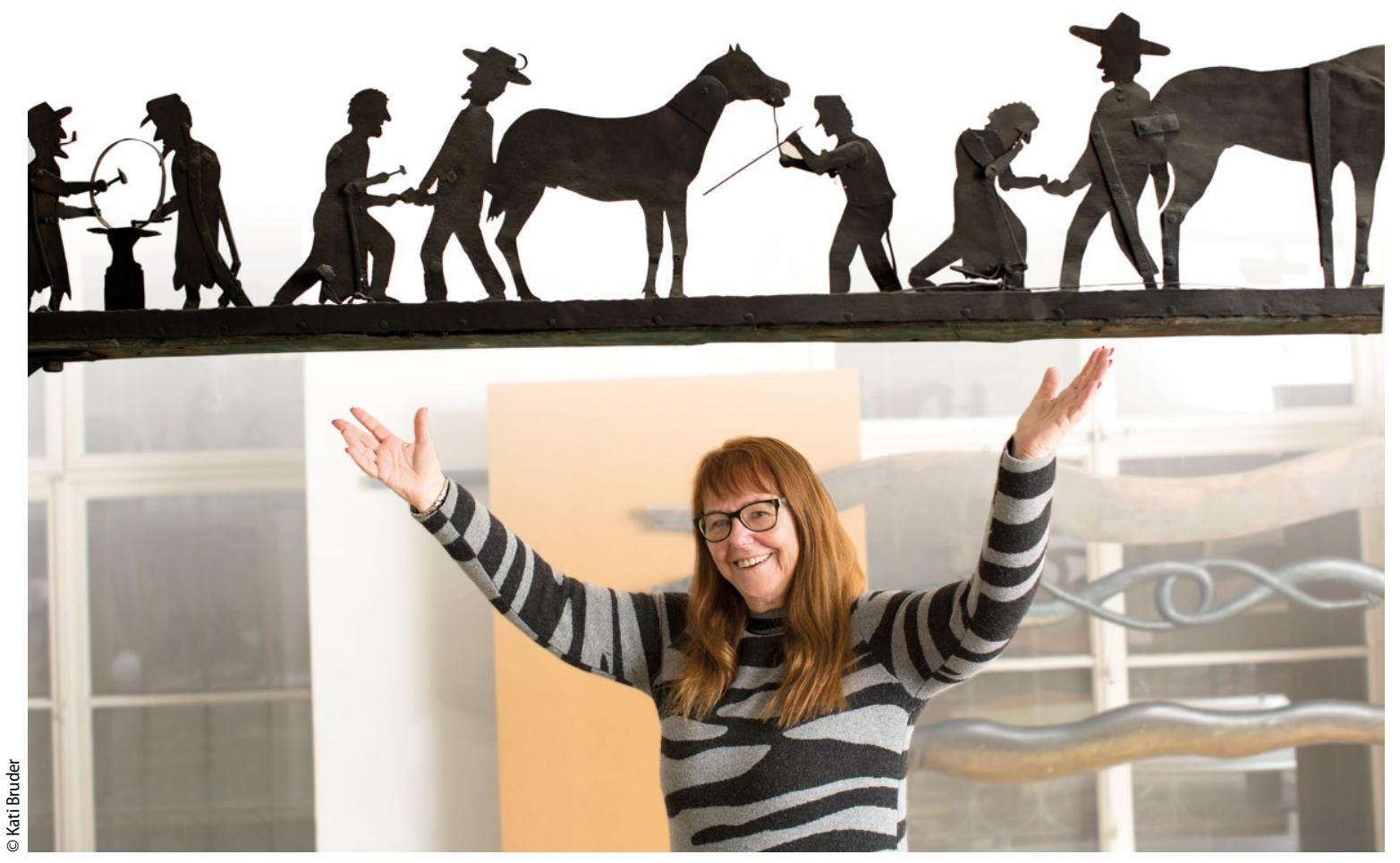

$\Delta$ Dr. Angelika Rosenberger-Spitzy ist Fachärztin für Innere Medizin und Journalistin, war bis 1990 Medizinische Chefredakteurin der ÄrzteWoche, dann zwölf Jahre Ärztliche Direktorin des Geriatriezentrums am Wienerwald, Chefärztin des Fonds Soziales Wien und Seniorenbeauftragte der Stadt. Jetzt studiert Rosenberger in ihrer Pension Europäische Ethnologie 
einer Immobilität. Wenn gebrechliche Menschen in der Nacht stürzen, werden blaue Flecken und Blutergüsse mit dem zunehmenden Alter immer dramatischer, weil die Blutgefäße immer poröser werden. Die Immobilität führt zu Muskelschwäche und der Muskelschwund steigert die Frailty. Umgekehrt führt die Supplementierung mit Vitamin D zu mehr Muskelkraft, zu mehr Fitness und Wohlbefinden. Patienten mit einem guten Vitamin D-Spiegel geht es einfach besser.

Schützt Vitamin D auch vor Infektionen?

Prof. Gerald Ohrenberger hat im Haus der Barmherzigkeit Patienten mit guten Vitamin D-Spiegeln mit solchen verglichen, deren Spiegel unter $30 \mathrm{nmol} / \mathrm{L}$ lagen. In der Vitamin D-Gruppe hatten $18 \%$ Antibiotika-pflichtigen Infekte. Die Gruppe mit schwächeren Vitamin DSpiegeln hatte zum Vergleich $82 \%$ - also sehr viel mehr - Infekte mit Antibiotika. Diese Daten sprechen dafür, dass der Antibiotika-Verbrauch in der Geriatrie durch eine Vitamin D-Prophylaxe wesentlich eingeschränkt werden kann.

Wirkt Vitamin D auch auf Krebszellen?

Mehrere Studien haben gezeigt, dass Nahrungsergänzungsmittel mit subtherapeutisch dosiertem Vitamin D keine messbare Wirkung zeigen. Wirksam sind erst höhere Dosierungen. Einige Länder wie z.B. Finnland haben sich entschlossen, Lebensmittel wie die Milch mit Vitamin D anzureichern. Das ist eine radikale Methode, die die individuellen Unterschiede zwischen den Menschen vernachlässigt. Aber offenbar haben die Finnen ihren Patienten so rund $20 \%$ der bisherigen Krebserkrankungen erspart. Das könnte zu einer Revolution in der Onkologie führen.
Hilft Vitamin D auch bei Covid-19?

Für diese Frage fehlen gute Studien. Eine Publikation weist allerdings darauf hin, dass bei ausreichendem Vitamin D-Spiegel schwere Verläufe seltener eintreten.

Gibt es geeignete Medikamente als Vitamin D-Supplemente?

Leider gibt es viel Unfug. Nahrungsergänzungsmittel enthalten in der Regel viel zu wenig Vitamin D für chronisch Kranke oder alte Menschen. Manche machen zwar Werbung damit, verschweigen aber die (geringe) Inhaltsmenge. Besser sind die zugelassenen Arzneimittel. Wenn die Patienten kein klares Briefing erhalten, tendieren sie allerdings mit den traditionellen Tropfen zu geringen Dosierungen. Eine Studie im Haus der Barmherzigkeit hat gezeigt, dass nur rund die Hälfte der Patienten mit Tropfen zurechtkommen. Die Gesundheitsbehörden haben deshalb ein hoch dosiertes Präparat mit 20.000 I.E. zugelassen. Diese Dekristolmin-Weichkapseln werden bei Vitamin D-Mangel erst 14 Tage lang täglich eingenommen um die Speicher in der Leber aufzufüllen. Anschließend genügt 1 Kapsel pro Woche, das ist eine erfreuliche Vereinfachung. Bei extremen Mangelerscheinungen, wie sie oft verschleierte arabische Frauen, chronisch Kranke oder Bettlägrige zeigen, sollte die Aufbauphase von 14Tagen auf 4 Wochen verlängert werden, bevor man auf den wöchentlichen Rhythmus umstellt. Weil es bei der Resorption auch individuelle Unterschiede geben kann, ist danach die Evaluierung der Therapie durch den Blutbefund notwendig. Der Hausarzt wird so zum Manager des Vitamin D.

\section{Ein Resumee?}

Für die Langzeittherapie sollte der Arzt nicht nur die richtigen Kapseln verordnen, sondern auch täglich Morgenturnen bei Fit mit Philipp

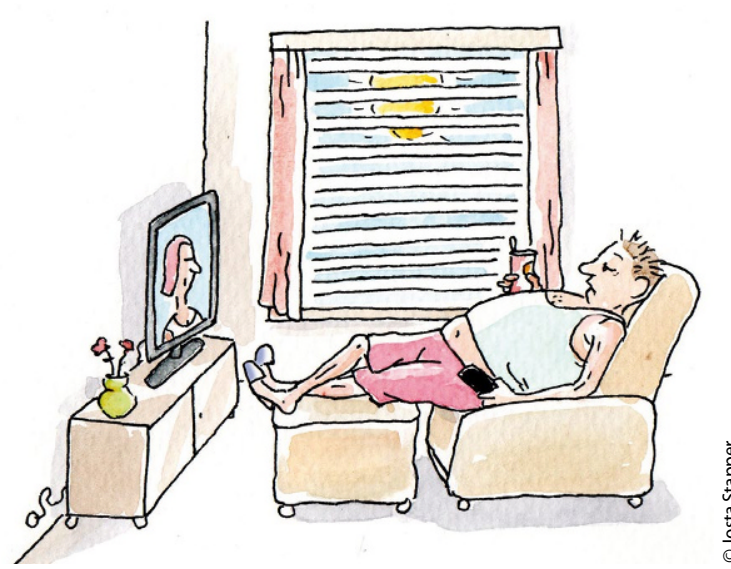

$\Delta$ Auf dem Weg zur Muskelschwäche

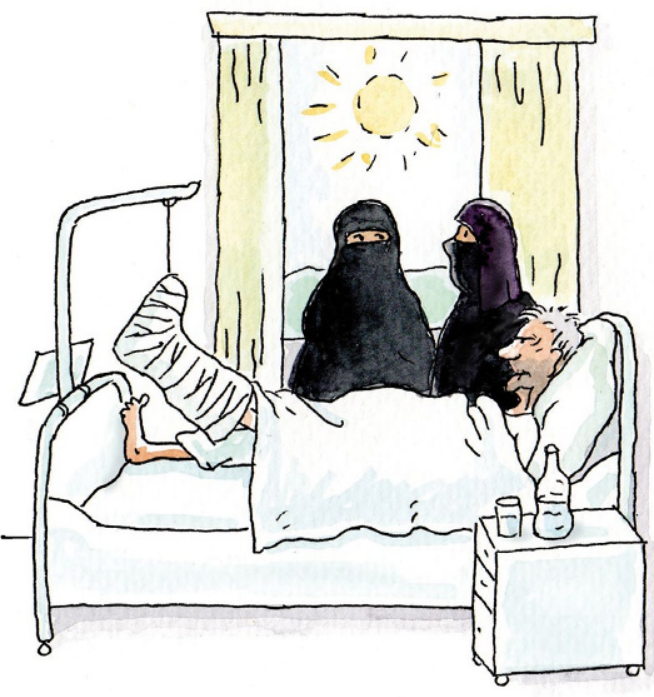

A $3 \times$ massiver Vitamin-D-Mangel

und eine halbe Stunde Spazierengehen in der Natur.

\section{Das Gespräch führte Toman Rom, MedNews}

Hinweis des Verlags. Der Verlag bleibt in Hinblick auf geografische Zuordnungen und Gebietsbezeichnungen in veröffentlichten Karten und Institutsadressen neutral.

rheuma plus $2021 \cdot 20: 54-55$ https://doi.org/10.1007/s12688021-00422-1

(c) Springer-Verlag GmbH Austria, ein Teil von Springer Nature 2021 